\title{
GULSTONIAN LECTURES
}

ON

\section{THE HEAT OF THE BODY. \\ DELIVERED AT \\ The Royal College of Physicians, London, MARCH I87I.}

By SAMUEL J. GEE, M.D., F.R.C.P., Assistant-Physician to St. Bartholomew's Hospital and to the Hospital for Sick Children.

\section{LECTURE II.-Concluded.}

WE will now proceed to the opposite condition-of the body being exposed to a temperature lower than itself. This is, indeed, the natural state of things, to which all that we have said before concerning the generation and regulation of heat has direct reference. In this place, however, we will consider the effect of great abstraction of heat from the surface of the body.

Rabbits can be cooled down to $48 \mathrm{deg}$. Fahr. before they die, if, at the same time, artificial respiration be kept up ; at this low temperature the animals still feel and move. Rabbits cooled down to 64 deg. cannot recover upon being brought into a warm atmosphere, unless artificial respiration be kept up; under the two conditions of external warmth and artificial respiration, the creatures recover even when their temperature has fallen to 64 deg. Rabbits, not cooled below 77 deg., recover by external warmth alone, without artificial respiration. (Walther.)

The effect of similar reduction of temperature in the human being is quite unknown; death by cold is so rare an occurrence compared with death by heat. But what if the external cold be less extreme or less prolonged in operation? Generally speaking, temporary cooling of the surface of the body causes the temperature of the blood to rise. A thermometer in the rectum of a drenched puppy continues to rise so long as evaporation goes on from the surface of the body. When the quadruped is brought, so far as his skin is concerned, into a state like that of man, by being shorn, and then is wrapped in a wet linen cloth which admits of evaporation, the temperature of the rectum falls twentyseven degrees in the course of an hour. Now, in the human subject, moderate external cold produces both these effects; according to circumstances, the temperature of the body either rises or falls.

Senator took the temperature of the arm-pit of a man whilst in bed in the morning. Afterwards, the man rose, and remained naked in the bedroom. It was found that the body-heat was depressed sooner or later when the temperature of the atmosphere was allowed to fall below $80 \mathrm{deg}$. Reversely, the temperature of the body rose when the heat of the room was greater than that of the blood; thereby proving what we asserted before, that it is only within limits of $18 \mathrm{deg}$. or so, say from 80 to $98.5 \mathrm{deg}$, , that the body can maintain its constant temperature for any length of time without voluntary external aid.

Next let us inquire-What is the effect upon the naked body of an external temperature below 80 deg. ? Senator, by his experiments, found that in a room below $80 \mathrm{deg}$. the temperature of the axilla always rises at first, and rises so much the more and so much the more rapidly, the lower the temperature of the room, within certain limits. The greatest elevation observed amounted to nearly one degree, after twenty minutes' exposure in a room of $60 \mathrm{deg}$. To this primary elevation of temperature succeeds a gradual fall, which is not broken by any fresh elevation so long as the experiment can be carried on. At first, however, this fall consists simply in a return to the temperature noted at the beginning of the experiment. Thus in a room at $57 \mathrm{deg}$., the body-heat at first was $97.9 \mathrm{deg}$., in thirty-five minutes it rose to 98.5 deg., in twenty-five minutes more it fell to what it was at first (97.9 deg.), and it continued to fall, reaching $97.5 \mathrm{deg}$. in fifteen minutes longer, a temperature which was accompanied by violent shivering and blueness of the skin, such as rendered it necessary to stop the experiment. And yet the body-heat was at 97.5 in the axilla.

A cold bath produces results which are precisely similar, except that cold water abstracts heat much more rapidly than cold air. For example : a man in a bath of $77 \mathrm{deg}$. will, as a rule, rise a little in temperature during the first quarter of an hour ; but, after this temporary rise, a fall sets in which is unbroken so long as the bath is continued. That the temperature rises in a cold bath was noted by Pickel in a book published so long ago as I788. But, during the last ten years, the influ- ence of cold baths has been very carefully studied, especially by Liebermeister of Basel.

The fact of the primary rise of temperature is admitted by all ; but how the fact is to be explained has led to great dispute. Two parties have been formed. Liebermeister and his party hold that the rise in temperature indicates an increased generation of heat in the body, for the purpose of counteracting the increased loss of heat, and by virtue of the power which the body possesses of spontaneously regulating its own temperature. On the other hand, Jürgensen and Senator deny that the body has any power of generating an increased amount of heat sufficient to regulate the temperature of the body under the condition of active abstraction of heat from the surface. The temperature of the internal parts rises under the influence of a cold bath, because, say they, the contraction of the blood-vessels of the skin prevents the entry of blood into them; and the result is that, between the internal parts and the cold water, there is a layer of ill-conducting tissue, which is constantly waxing thicker and thicker; so that, in fact, the body loses, not more, but less heat than natural. Meantime, the ordinary amount of heat is being produced; ergo, the temperature of the blood and internal organs rises.

After the bath, the temperature continues to fall. How is this to be explained? Jürgensen supposes that the blood, as it re-enters the cold skin, thereby becomes itself cooled to such a degree as to depress the temperature of the whole mass of blood. But Liebermeister, regarding the cold skin as a stimulus to increased generation of heat, conceives that, when the warm blood enters the skin again, the production of heat ceases to be unnaturally great, and falls to the usual standard.

Liebermeister endeavoured to support his opinion-that the elevated temperature indicates increased generation of heat-by some rather rough calorimetric observations. But, quite recently, Gildemeister has published important evidence in favour of Liebermeister's view. That is to say, the amount of carbonic acid expired is found to be greatly increased at the time when the temperature rises. And this increased exhalation of carbonic acid must correspond to increased chemical combination. A man, dressed in the usual way, expired 15.3 grammes of carbonic acid in half an hour; during the next half hour, he was stripped and washed with ice-cold water, the carbonic acid was nearly doubled in amount, namely 27.8 grammes; dressed again for half an hour, the quantity fell to its former amount, 15. I grammes; cooling the skin as before raised it to 24.9 grammes; and the man, dressed again for another half hour, expired the usual quantity of carbonic acid, I5.6 grammes. Gildemeister also found that the increase in the expired carbonic acid was directly proportionate to the coldness of the bath. A man, who exhaled 13.2 grammes of carbonic acid per half hour under ordinary circumstances, in a bath of 91 deg. Fahr. exhaled 14.8 grammes; in a bath of $78 \mathrm{deg}$. Fahr., exhaled 22.5 gramımes; at $68 \mathrm{deg}$. Fahr., 38.9 grammes; and at 65 deg. Fahr., 39 grammes, or exactly three times the normal quantity.

I think that there can be no doubt that the body does possess the power of spontaneously adjusting the supply of heat to the demand, within certain limits. And the conditions of an increased formation of heat are present in the case supposed. The viscera and the muscles contain more blood than usual, and these are the great calorific organs; moreover, the respiration is proportionately frequent and deep. But no doubt there is truth in what Liebermeister's opponents say concern. ing the protective influence of the cooled skin. Indeed, as Senator observes, the skin is a sort of reservoir for the blood, whereby the internal parts escape material change of temperature. For the body's powers of liberating heat are very limited ; if the degree or the duration of the withdrawal of heat be too great, the body is not able to counteract the loss; the result being that the internal parts, also, are cooled. The limit seems to differ in different individuals, and also in the same individual at different times; an abstraction of heat which at one time produces lowering of the temperature of the blood, at another time raises its temperature.

Jürgensen found that cold baths repeated daily, day after day, produced a very curious effect upon the temperature of the body. The time during which the temperature was raised increased with each bath, and, more than this, the mean daily temperature also rose. This change in the heat-economy of the body was so prolonged as to influence the action of the next bath; and thus the cooling power of the baths became progressively less and less, until it was reduced to none at all. For example : a man, after a number of daily baths at about 50 deg., took one of $48 \mathrm{deg}$. for twenty-five minutes; during this last bath, his temperature remained at $99.6 \mathrm{deg}$. Jürgensen admits that this points to increased production of heat. Dogs exposed to cold are able to replace a loss of 9 or 10 deg., affecting the whole body, in an hour or less.

I need not stay to remark upon the important bearing which these 
facts have upon the therapeutics of what is called the water-cure. Local abstraction of heat, by hip-baths or otherwise, produces the same effect as full-length baths.

The influence of baths, hot or cold, upon the temperature of the body at large, depencis (as we have seen) to a great extent upon the effect which they have upon the vascularity of the skin. The cooling power of cold baths is much diminished by the contraction of the superficial vessels of the body, whereby they are emptied of their blood. On the other hand, the abundant evaporation from the surface, which goes on in a hot-air bath, is insufficient to counteract the heating effect of the bath, by reason of the hyperæmic state of skin which greatly favours absorption of the external heat. Now let us put the opposite condition : the skin, unnaturally full of blood, exposed to a low temperature. These conditions are fulfilled when an animal, whose skin has been shaven and varnished, is left, without any covering, in the ordinary temperature of the air. It has long been known that, under these circumstances, the animal quickly died; and, naturally enough, those who first noted the fact supposed that some noxious elements of the perspiration were retained in the body. If it were a case of simple suppression of sweat, the temperature of the body should rise. Far from this being the case, the heat is so rapidly lost, that the death of the animal is undoubtedly due to the depression of temperature. Even supposing that the amount of blood in the skin remained the same before and after the varnishing, even then the varnishing would increase the loss of heat two-and-a-half-fold; in addition to this, the varnished skin becomes hyperæmic, just as if its vaso-motor nerves were paralysed. And, by way of an experimentum crucis, Krieger has succeeded in keeping varnished rabbits alive and active for five days, by surrounding them with an atmosphere of from 90 to $95 \mathrm{deg}$.

Bearing these facts in mind, it becomes an interesting question whether the depressed temperature which is observed soon after an extensive burn, may not be partly or chiefly due to the hyperæmia of the skin. In one case of extensive burn, Billroth found the temperature of the arm-pit to be only $91.5 \mathrm{deg}$. Fahr. soon after the accident ; the patient was put into a warm bath of from 95 to 104 deg.; in two hours the temperature of the axilla rose to $99 \mathrm{deg}$., then gradually sank, but rose again shortly before death to $102 \mathrm{deg}$.

We have now discussed two divisions of our subject. First, we examined the means by which the body gains and loses heat. In the second place, we considered the result of these contrary processes in the healthy state-that is to say, the natural temperature of the body. Let us pass on to investigate the deviations from the natural standard of body-heat-temperatures which are unnatural.

By the beat of the body we mean the heat of the blood. And we judge of the heat of the blood by the heat of the axilla or of the rectum. We will assume it as being generally true, that a temperature above $99.5 \mathrm{deg}$. in the axilla, or above Ior deg. in the rectum, is doubtless unnaturally high ; and that a temperature below $96 \mathrm{deg}$. in the axilla, or $98 \mathrm{deg}$. in the rectum, is unnaturally low. Thus we have two great types of unnatural temperatures. However, I shall not specially ask your attention except to the case of the general heat of the body being unnaturally high - the $\pi v \rho, \pi v \rho \in \tau o s, \pi v \rho \in \xi i s$ of Hippocrates.

And here we are met, at the very outset, by an opinion that, in what we call pyrexia, the temperature of the blood is not really raised; an opinion which has been rendered possible by observations such as made by John Hunter, who noticed that the difference between pyretic and apyretic temperatures became less and less marked the more deeply seated the parts of the body examined. Bernard's famous experiment of dividing the sympathetic nerve (causing thereby great elevation of temperature in the skin of the parts concerned, as compared with the corresponding parts on the opposite side of the body) gave a new impulse to this hypothesis ; especially when taken along with an assertion made by the same physiologist, that the temperature of the liver is sometimes as high as $106 \mathrm{deg}$. in health. Putting these two statements together, Marey has been able to maintain that pyrexia is due merely to a loss of vascular tone; whence dilatation of the vessels, and diminution of the resistance which the small arteries afford to the blood-stream. Hence diminished pressure in the arterial system, and increased rapidity of the circulation. Hence, lastly, equalisation of internal and superficial temperatures. So that, to quote Marey's words, "The elevation of temperature under the influence of fever consists much more in a levelling up of the temperature in different parts of the economy than in an actual heating of the body." Doubtless there is in this view a certain amount of truth which we must not reject ; but the hypothesis, as Marey puts it, is quite untenable. First, there is good reason to believe that Bernard rated the possible temperature of the liver in health too high, and that, in fact, it never exceeds ro4 deg.: so that all febrile temperatures above 104 deg. are inexplicable upon Marey's hypothesis.
Moreover, Jacobson has observed that the temperature of the liver rises with that of the rest of the body in dogs rendered artificially pyretic. And although there is no doubt about the dilatation of the small arteries in certain stages of fever-heat, there is no proof whatever that the diminished arterial pressure and increased rapidity of circulation are the causes, and not, as heretofore supposed, the effects of pyrexia.

The temperature of the blood, then, is really raised. This elevation of temperature obviously must be due, either to an increased production of heat, or to a diminished loss of heat, or to a combination of these causes. Let us consider each of these possible conditions separately.

When we discussed the physiology of animal heat, we saw that it had been explained by three theories : the theory of innate heat, the mechanical theory, and the chemical theory. Now, a theory competent to explain the existence of animal heat in general, might be reasonably expected to be able to explain the existence of animal heat in particular. Accordingly, we find that the pathology of animal heat has always been consonant with the current physiology of the day. The earliest, or innate heat doctrine, hardly deserves the name of theory. To say that pyrexia is an increase of the calidum innatum may be a definition, but is not an explanation. When the mechanical theory took the place of the defunct calidum innatum, the theory of fever became mechanical : and as with the physiology of the mechanical theory, so with its pathology ; it will be most conveniently deferred until we have examined the third and latest theory of pyrexia - the chemical.

The chemical theory of animal heat was, we know, established by Lavoisier in the last quarter of the last century. But the doctrine of unnatural animal heat was influenced in no wise by Lavoisier's great discovery. This is a very remarkable fact. If the natural heat of the body be due to a certain amount of chemical combination, it does not seem to be a very great stride to the corollary, that increased heat is due to increased combination. And yet this step was not taken for half a century ; a fact which is probably to be explained by the absorbing interest which was taken in the study of morbid anatomy. Morgagni had preceded Lavoisier, but the Baillies and Laennecs who appeared after him drew all men's minds to the study of pathological structure ; pathological physiology is a growth of later date. The natural result of this state of things was the appearance of such men as Broussais and Clendinning, who talked of physiological medicine, but endeavoured to explain fever by mere anatomy. Fever became a symptom of a local lesion. Volumes upon volumes appeared concerning the nature of in. flammation; nobody seemed to remember that there was such a state as pyrexia worthy of attention. The great methods of physical examination which were discovered at the same time referred wholly to local anatomical changes (auscultation and percussion); whereas the thermometer was passed by.

I will not further develope this historical sketch of events which follow one another with a fatal order equal to that of the layers of a geological section. - Let us come at once to the time when what Lavoisier had done for animal heat in general another great chemist did for preternatural animal heat in particular. I allude to Liebig. $\mathrm{He}$ it was who, thirty years ago, first laid down the proposition that when (in consequence of morbid metamorphosis of the living parts of the body), a greater amount of force is set free than is necessary to the normal organic movements, straightway there ensues increase of some or of all of the organic functions, and, as one result thereof, elevated temperature. Certainly this is simply an application of the old aphorism, "Quæ faciunt, in homine sano, actiones sanas, eadem, in ægroto, morbosas." Those chemical processes which beget a normal temperature, beget, also, an abnormal temperature.

But yet we may not leap to the conclusion that augmented production of heat is the necessary antecedent of pyrexia ; might it not be due to retention of heat in consequence of diminished loss? In fact, the latter alternative has been, and still is, upheld by some pathologists ; so that you will not deem it needless to ask what are the proofs that the amount of heat begotten by the pyretic body really is increased. And proof will be forthcoming, if we find that the temperature of the body is greater than natural whilst the loss is not less. What, then, is the amount of heat given off by the pyretic body? This is obviously a question of calorimetry. But calorimetry is always difficult, and calorimetry of a febrile person must be very difficult. Nevertheless, quite lately Leyden has overcome these difficulties. He encloses the leg alone in a calorimeter. And it is a certain kind of witness to the trustworthiness of Leyden's observations, that the total amount of heat given off by the surface of the healthy body, calculated from his data, agrees closely with the estimate of Helmholtz. Leyden's experiments were made chiefly upon a patient with relapsing fever. He found that the heat given up to the calorimeter, during the period of pyrexia, was never below the standard of health. But the skin did not lose heat in proportion to its temperature alone; indeed, as we might have expected, 
the skin lost heat rather in proportion to the evaporation of sweat from the surface. In the cold stage of fever, the amount of heat lost is not diminished, but is about the same as the standard of health. The loss, I say, is not diminished ; the temperature is rising greatly; therefore, the production of heat is greatly increased. Next, in the hot stage, the heat lost from the skin is about double the normal. Next, in the sweating stage, the heat lost is about thrice the normal amount; it was so even in a case in which the temperature fell nearly two degrees in an hour. Now, a sweating skin would certainly not radiate more heat than a hot dry skin of higher temperature ; so that it seems to follow as a necessary consequence that the loss of heat is chiefly dependent upon the evaporation of sweat. It is not that, during the hot stage, there is a diminished loss of heat from the surface (as has been supposed by some), for the loss is actually doubled; but, although doubled, it does not suffice to carry off the excess of heat generated; there is absolute increase of the cutaneous cooling, but relative diminution of it ; the balancing powers are at fault. Lastly, after the crisis, the loss of heat falls to below the normal. These observations of Leyden's agree, in all important points, with the less exact experiments of Liebermeister and others, made upon fever-patients put into baths of cold water. Let us accept it, then, as proved, that the increased temperature of the pyretic body indicates increased production of heat, inasmuch as there certainly is no diminished loss of heat so long as the temperature of the body remains above the normal.

The production of heat is increased; and this fact, according to the chemical theory, involves increased combustion of the constituents of the body. Increased combustion would be attended by increase in the quantity of excreted products of combustion. Is this found to be the case by actual experiment? Let us ask this question with regard to the two most important excreta of the body, those which represent the consumed carbon and nitrogen-I mean carbonic acid and urea. And we will take the carbonic acid first, because it is a much more significant and trustworthy product of combustion than is the urea. The weight of carbonic acid given off from the body exceeds that of urea twenty times.

The quantitative estimation of the carbonic acid expired by feverpatients is not easy. The earlier experiments of Malcolm seemed to show that, in typhus-fever, the relative quantity of carbonic acid expired was diminished; that is to say, the expired air of fever-patients contained a smaller percentage of carbonic acid than the breath of healthy persons. Lehmann and others have made observations upon small animals, which seem to show an absolute diminution of carbonic acid expired during the febrile state. But these experiments are not very conclusive. For a valuable series of observations upon this point, we are again indebted to Professor Leyden, of Königsberg. Leyden's experiments were made chiefly upon cases of typhus fever, relapsing fever, and pneumonia. He found that Malcolm's statement was correct; there is a small diminution of the carbonic acid relatively to the total quantity of air expired; a proportion of about nine in the febrile, to ten in the non-febrile state. But then the respiration is very much increased in frequency during fever, so that the quantity of air expired is half or three-fourths greater than the normal. Putting these facts together, it appears that the absolute quantity of carbonic acid expired during the febrile state is considerably increased, bearing a ratio to the non-febrile state of ahout one-and-a-half to one. It will be understood that the febrile and non-febrile states were compared under circumstances which were otherwise the same-namely, a slender diet and rest in bed. Liebermeister has made experiments of a similar kind upon two cases of ague ; the results obtained agree with those of Leyden. During the cold stage, the carbonic acid was increased in quantity by nearly 150 per cent.; in other words, it was nearly two-and-a-half times the normal amount. During the hot stage, the increase was much less, from 19 to 31 per cent. During the sweating stage, the increase was little or none. Now it is highly probable that, in all stages of fever, the quantity of carbonic acid expired affords the best measure of the whole tissue-metamorphoses which result in the liberation of heat. If this be so, we may draw the following conclusions: that in the cold stage of fevers the production of heat is greatly increased, whilst, as we have already seen, the loss remains stationary at the standard of health; that in the hot stage the production is increased (but to a less degree than in the cold stage), whilst the loss is increased also; and that in the sweating stage the production falls to the standard of health, whilst it is the loss which is greatly augmented.

Observations made upon the quantity of urea excreted by febrile patients are just as numerous as those concerning the carbonic acid are few. There is, indeed, one drawback to these urea estimations, which have all been made, so far as I know, by Liebig's volumetric process, the results whereof are not altogether trustworthy, but still they are probably sufficiently near to the truth to deserve our attention. In the short fevers, such as ague and pneumonia, all observers, from Vogel down to those of the present day, agree that the quantity of excreted urea is increased during the pyretic state; the comparison being made upon the same person in the febrile and non-febrile states, under the same conditions as to food and rest in bed. Speaking generally, pyrexia increases the urea by one-half or three-fourths ; pretty much the same as the carbonic acid. And the experiments of Bartels, Senator, and Unruh show that the urea and uric acid are increased pari passu, provided the respiration be not interfered with.

This question now arises: Are the nitrogenous excreta of the urine increased proportionately to the temperature of the body? To this it is easy to give a distinct negative. In fact, no relationship at all can be established between the two conditions, nor, I think, is it difficult to see why this should be so. For, granted that the urea is proportional to the activity of combustion, the temperature certainly is not so. In the case of ague, it seems to me that the quantity of water excreted has more to do with the amount of urea present in the urine at different stages of the fever than any other single circumstance. For instance; during the sweating stage, the urine is usually much diminished in quantity, and in that case the urea is diminished too; after the crisis, both water and urea are excreted in excess. But sometimes the kidneys sweat at the same time as the skin ; the water of the urine is abundant, and so is the urea ; nor does there follow any epicritical excess of urea in the urine. Put now the case that, during the hot stage and the crisis, there is a falling off in the quantity of urea, and again an increase after the crisis : how are these facts to be explained? Let us bear in nind that the amount of heat generated after the crisis is less than natural, and yet, I say, the urea may be increased. Is there a retention of the excernenda during the hot and critical stages; and, if so, are these excernenda retained in the form in which they are excreted or in a less oxidised fr : $\mathrm{m}$ ? Or is the epicritical excess due to the absorption and combusti in of exudations such as are present in pneumonia, or such as Richard Volkmann and Steudener have found to accompany all fevers; namely, a great quantity of lymphoid corpuscles which are formed in the different tissues and organs, and which are reabsorbed during convalescence? At any rate, the body loses water with great rapidity during and shortly after the crisis-a fact testified by the loss of weight, and by the emaciation which becomes notable about that time.

In pyrexia, then, the quantity of urea excreted is increased-that is to say, the same increased combustion which liberates more heat than natural also produces more urea than natural. But increased combustion does not necessarily imply pyrexia. Let us now ask: Of the two conditions, elevation of temperature, or pyrexia, and increased oxidation of nitrogenous matters, as represented by the urea, which comes first? Now, the elevation of temperature during the cold stage of fever undoubtedly indicates increased generation of heat; this we have already seen. Well, then, in ague the rise of temperature is sometimes preceded by a great increase in the quantity of urea excreted - that is to say, the urea is augmented before the temperature. The same condition occurs, according to Naunyn, in the pyrectic state, produced by injecting putrilage into the subcutaneous tissue of dogs. In this experiment the temperature does not rise until two hours after the operation; but, during these two hours of natural temperature, the urea is increased in quantity above the normal (the water being at the same time diminished), a fact which seems to show that increased consump. tion of the body-tissues precedes the elevation of temperature. If this be so, the body must be able to dispel the excess of heat formed before the regulating function is involved, before the cold stage begins. Hereafter we shall have to discuss the question reversed, whether elevation of temperature does not produce increased consumption of tissue.

But when we pass on to consider the quantity of urea excreted during long fevers, the problem becomes much more complicated; appetite and digestion are so greatly impaired; nor are we able to keep such patients on their fever-diet after the fever is over, wherefore we are unable to obtain our usual standard of comparison.

\section{HYDRATE OF CHLORAL AS A CAUSE OF URTICARIA.}

IN June of last year I had occasion to give a patient (a stout middle. aged woman) a hypnotic. I ordered the following draught; viz., hydrate of chloral, 25 grains; simple syrup, a sufficiency; water, $1 \frac{1}{2}$ ounce. After taking it, the patient became the subject of extensive urticaria. I inquired very particularly whether she had partaken of any of the articles of diet sometimes producing the eruption, as oatmeal, shell-fish, etc. ; and was assured that she had not. I of course discontinued the chloral hydrate, at the same time being unwilling to give it the credit of producing the rash. Twenty-six days afterwards, I gave her a dose of ten grains, and in a short time the before mentioned effects were again produced.

S. Winter Fisher, M.D.

Clifton, March 387 I. 